\title{
Stage at diagnosis, clinicopathological and treatment patterns of breast cancer at Bugando Medical Centre in north-western Tanzania
}

JOSEPH B. MABULA ${ }^{1}$, MABULA D. MCHEMBE ${ }^{2}$, PHILLIPO L. CHALYA ${ }^{1 *}$, GEOFREY GIITI ${ }^{1}$, ALPHONCE B. CHANDIKA ${ }^{1}$, PETER RAMBAU ${ }^{1}$, NESTORY MASALU ${ }^{1}$ and JAPHET M. GILYOMA ${ }^{1}$

${ }^{1}$ Catholic University of Health and Allied Sciences-Bugando, Mwanza, Tanzania

${ }^{2}$ Muhimbili University of Health and Allied Sciences, Dar Es Salaam, Tanzania

\begin{abstract}
Breast cancer, although reported to be the commonest female malignancy worldwide has not been extensively studied in north-western Tanzania. The aim of this retrospective review was to describe in our setting, the stage at diagnosis, clinicopathological and treatment patterns among patients with breast cancer. Data were analyzed using SPSS software system. A total of 384 patients were studied. The median age was 45 years (range 21 to 78 years). The male to female ratio was 1: 46.8. Most of the patients were premenopausal $(63.8 \%)$ and presented late with advanced breast cancer disease. Majority of patients (63.0\%) presented with stage III disease. Lymph node and distant metastasis at the time of diagnosis was reported in $70.8 \%$ and $21.4 \%$ of patients, respectively. Invasive ductal carcinoma $(91.7 \%)$ was the most frequent histopathological type and most patients $(63.8 \%)$ had poorly differentiated tumour. Patients with tumour size greater than $6 \mathrm{~cm}$ had significantly high rate of lymph node metastasis $(P=0.001)$ and presence of necrosis within the tumour $(P=0.012)$ compared to patients with tumour size less than $6 \mathrm{~cm}$ in diameter. Patients younger than 45 years had significantly high rate of lymph node metastasis compared to the patients above this age $(P=0.011)$. Mastectomy was the main modality of treatment that was used in $99.5 \%$ of the patients. Adjuvant chemotherapy and radiotherapy was reported in $44.8 \%$ and $11.7 \%$ of patients, respectively. Hormonal therapy (tamoxifen) was given postoperatively to all patients. The overall five-year survival rate was $21.8 \%$. The age of patient at diagnosis, stage of disease, extent of lymph node involvement and histological grade were found to be independent predictors of overall survival rate $(P<0.001)$. Local recurrence was $17.7 \%$ and it was significantly related to the stage of disease $(P=0.003)$ and non-adherent to adjuvant therapy $(P=0.021)$. Breast cancer patients in this region are relatively young premenopausal women and mostly present late with advanced stage and high rate of lymph node metastasis. There is need to improve public enlightenment of breast cancer and set up screening centres to encourage early presentations.
\end{abstract}

Keywords: breast cancer, stage, diagnosis, clinicopathology, treatment, Tanzania

\section{Introduction}

Breast cancer is one of the commonest malignant tumours in the world and is one of the leading causes of death due to cancer in women (Abdallah et al, 1999; Christobel \& Baun, 2000; Okobia \& Osiime, 2001; Ikpatt et al., 2002; Ohanaka \& Ofoegbu, 2002). The incidence rates of breast cancer show considerable global variation being higher in developed countries than in developing countries (Vorobiof et al., 2001; Ikpatt et al., 2002). In Africa, breast cancer has overtaken cervical cancer as the commonest malignancy affecting women and the incidence rates appear to be rising (Vorobiof et al., 2001; Omar et al., 2003). In Tanzania, breast cancer constitutes the second leading cause of cancer incidence and mortality for women (Ngoma \&

\footnotetext{
* Correspondence: Phillipo L. Chalya; E-mail: drphillipoleo@yahoo.com
} 
Mtango, 2002), and the lifetime risk for developing breast cancer in Tanzania like in most developing countries is approximately 1 in 20 (Parkin, 2003).

The increase in the incidence of breast cancer in developing countries can be attributed to changes in the demography, socio-economic parameters, epidemiologic risk factors, better reporting and awareness of the disease (Parkin et al., 2005; Adesunkanmi et al., 2006). While mortality rates are declining in the developed world as a result of early diagnosis, screening, and improved cancer treatment programmes, the converse is true in the developing world (Hisham \&Yip, 2004; Parkin et al., 2005; Adesunkanmi et al., 2006).

One major determinant whether a cure for breast cancer will be possible or not is the stage at which the patient presents. Several factors determine the stage at which patients with breast cancer present to the hospital. Some of such factors include educational status, financial capability, occupation, location, presence of health care facilities, etc. However, a common denominator of these factors is the level of awareness and attitude toward breast cancer. For those patients that are aware about the disease and have the right attitude toward it, there is an increased chance that they will present early for treatment, while the contrary will be the case for those patients who may not be aware of breast cancer and also have wrong attitude toward the disease (Okobia et al., 2006).

The clinical stage of the disease at presentation is important for the outcome of the patient with breast cancer. However, in most African countries including Tanzania, patients present late with advanced stage of the disease and only palliative care is possible (Amir et al., 1994; Ngoma \& Mtango, 2002; Rambau et al., 2011). This can be explained by delay in seeking medical services and poor medical service with no screening programmes for breast cancer. The staging of the patient in conjunction with histological grade can be used to determine the prognosis of the patient. Whereas stage I disease has about $96 \% 5$-year survival rate, this can be as low as 18\% 5-year survival rate in stage IV (Elston \& Ellis, 1991; Henson et al, 1991; Elston et al., 2005; Rambau et al, 2011).

The management of breast cancer posses a great challenge in resource limited societies as found in Africa. Late presentation of the disease, lack of adequate mammography screening programmes, preponderance of younger pre-menopausal patients and a high morbidity and mortality are among the hallmarks of the disease in Africa (Vorobiof et al., 2001; Adesunkanmi et al., 2006). The results of prevention of breast cancer or breast cancer cure have not been very encouraging more so in the developing countries (Gakwaya et al., 2008). The present day knowledge of this disease does not have any effective primary prevention. It is thus imperative that efforts should be made to detect the disease in its early stages. Mammography has been found to be useful but it is not feasible for mass screening in most developing countries as there is a limited number of units accessible to the general public (Ngoma \& Mtango, 2002). Public education towards Breast Self Examination should be propagated because it is practical and affordable (Ngoma \& Ngoma, 2004).

The current treatment of breast cancer includes surgery, chemotherapy, radiotherapy, hormonal therapy and targeted therapy using monoclonal antibodies like Bevacuzumab and Trastuzumab as well as palliative and supportive care in advanced cases (Elumelu et al., 2011). The treatment of breast cancer in sub-Saharan Africa is largely limited to surgery because of advanced stage at presentation and limited access to diagnostic imaging as well as adjuvant 
therapy. Mastectomy is, therefore, the treatment of choice for the majority of cases (Vorobiof et al., 2001; Omar et al., 2003; Adesunkanmi et al., 2006).The outcome of treatment of breast cancer in our environment has been poor because the majority of these patients present late to the hospital with advanced stage. This is partly due to paucity of local data regarding this condition and lack of community awareness on the importance of early reporting to hospital for early diagnosis and treatment.

Despite the effort made by the Medical Women Association of Tanzania (MEWATA) on breast cancer awareness and screening campaign in most parts of Tanzania, breast cancer continues to be a major cause of morbidity and mortality and majority of these patients still present late with advanced stage (Ngoma \& Mtango, 2002; Rambau et al., 2011). This study was therefore, undertaken to describe the stage at presentation, clinicopathological and treatment patterns in a consultant hospital in north-western Tanzania.

\section{Methods and Patients}

\section{Study area and design}

This was a retrospective study of histologically confirmed breast cancer patients seen at the department of Surgery of Bugando Medical Centre (BMC) over a period of 10-years (January 2002 and December 2011). BMC is a consultant, tertiary care and teaching hospital for the Catholic University of Health and Allied Sciences-Bugando (CUHAS-Bugando) and has 1000 beds. The hospital is located in Mwanza in north-western Tanzania and serves as a referral centre for tertiary specialist care for a catchment population of approximately 13 million people from Mwanza, Mara, Kagera, Shinyanga, Tabora and Kigoma.

The hospital has a newly established Oncology Department which provides care for all patients with histopathologically proven cancers including skin cancers. However, the department does not currently provide radiotherapy services. As a result patients requiring this modality of treatment have to travel long distances to receive radiotherapy elsewhere.

\section{Study population}

The subjects of this study included all patients who presented to BMC with histologically confirmed breast cancer during the period under review. Patients with incomplete data were excluded from the study. The details of patients were obtained using data derived prospectively from BMC medical record database, 2002-2011 and also from patients' files kept in the Medical Record Department, the surgical wards, operating theatre and histopathology laboratory. The study variables included demographic data (age, sex), parity, family history of breast cancer, marital status, menopausal status, duration of illness, anatomical side and quadrant, tumour size, pathological nodal status, clinical stage, histopathological type and grade, treatment modalities, outcome and follow up. The outcome variables in this study were survival and local recurrence. This information was collected using a pre-formed questionnaire.

\section{Determination of histological type and grade}

Determination of histological type and grade was established by Modified Bloom-Richardson score system which scores for tubular formation, nuclear pleomorphic and mitotic rate within 
tumour cells. Other tumour morphological features like presence of necrosis, and involvement of the skin was evaluated. The clinical stage of the disease was assigned to each patient by using TNM (AJCC Cancer Staging Manual). This is a staging system which is expression of anatomical extent of disease based on extent of primary tumour $(\mathrm{T})$, absence or presence of and extent of regional lymph node metastasis $(\mathrm{N})$ and absence or presence of distant metastasis. Other investigations performed include full blood count, serum urea and electrolytes and liver function test. Chest radiograph and abdomino-pelvic ultrasound and CT scan were performed to rule out distant metastasis.

\section{Treatment modalities and follow up of patients}

Treatment was based on the tumour stage and patient's performance status. Patients with early or locally advanced disease were treated with modified radical mastectomy (MRM). Patients with metastatic disease were offered simple or toilet mastectomy \pm axillary clearance as indicated. Adjuvant chemotherapy and radiotherapy were given postoperatively. Patients requiring radiotherapy were referred to Ocean Road Cancer Institute in Dar es Salaam. Hormonal therapy using tamoxifen was offered to all patients at a daily dose of $20 \mathrm{mg}$ for five years as oestrogen receptors and progesterone receptors were not tested. Follow-up visits were scheduled every 3 months for 2 years and every six months thereafter for a period of five years or death. Survival analysis was carried out with survival defined as the time between the date of commencement of treatment and the date of last follow-up or death (Sant et al., 2004).

\section{Data analysis}

Statistical data analysis was done using SPSS software version 17.0 (SPSS, Inc, Chicago, IL, USA). Data was summarized in form of proportions and frequency tables for categorical variables whereas continuous variables were summarized using median and ranges. Chisquare $\left(\chi^{2}\right)$ test was used to test for significance of associations between the predictor and outcome variables in the categorical variables) whereas independent student $t$-test was used in continuous variables. Odds ratio (O.R.) was calculated to test for strength of association between predictor variables. Multivariate logistic regression analysis was used to determine predictor variables that are associated with outcome. Significance was defined as a p-value of less than 0.05 .

\section{Ethical consideration}

Ethical approval to conduct the study was obtained from the CUHAS/BMC Joint Institutional Ethic Review Committee before the commencement of the study.

\section{Results}

\section{Characteristics of patients}

During the study period, a total of 399 patients with histopathologically confirmed primary breast cancer were admitted to BMC. Of these, 15 patients were excluded from the study due to incomplete data, leading to a final study population of 384 breast cancer patients. The patients' ages at the time of diagnosis ranged from 21 to 78 years (median= 45 years). The modal age 
group was 31-40 years accounting for $49.7 \%$ of cases. The majority of patients, $215(56.0 \%)$ were young than 40 years. Eight (2.1\%) patients were males and $376(97.9 \%)$ were females with a male to female ratio of 1: 46.8. Most of the patients were married (67.7\%). Family history of breast cancer was documented in only $2.3 \%$ of cases. Out of 376 female patients, $240(63.8 \%)$ were pre-menopausal (Table 1).

Table 1: Characteristics of patients with breast cancer at BMC

\begin{tabular}{llll}
\hline Patient characteristics & Response & Number of patients & Percentages \\
\hline Age in years & $<30$ & 24 & 6.3 \\
& $31-40$ & 191 & 49.7 \\
& $41-50$ & 90 & 23.4 \\
Sex & $51-60$ & 63 & 16.4 \\
Parity & $>60$ & 16 & 4.2 \\
& Males & 8 & 2.1 \\
& Females & 176 & 97.9 \\
& Nulliparous & 57 & 14.8 \\
Family history of breast cancer & Multiparous & 200 & 52.1 \\
& Grandmultiparous & 79 & 20.6 \\
& Not documented & 48 & 12.5 \\
Marital status & No & 14 & 3.6 \\
& Not documented & 306 & 79.7 \\
& Married & 64 & 16.7 \\
& Single & 260 & 67.7 \\
Menopausal status & Divorced/widowed/separated & 44 & 11.5 \\
& Not documented & 68 & 17.7 \\
& Pre-menopausal & 12 & 3.1 \\
& Post-menopausal & 240 & 63.8 \\
\hline
\end{tabular}

\section{Clinicopathological pattern}

The duration before presentation of the initial tumour ranged from 1 to 36 months with a mean of $11.4 \pm 5.2$ months. The majority of patients (54.4\%) presented to the BMC between 6 to 12 months of onset of symptoms. Three hundred and eighty-two (99.5\%) patients presented with a lump followed by ulceration/erythema and nipple retraction in 214 (55.7\%) and 204 (55.1\%) patients respectively. Nipple discharge and arm swelling was documented in $84(21.9 \%)$ and 12 (3.1\%), patients respectively. Right breast was involved in 198 (51.6\%) patients. Upper outer (lateral) quadrant was commonly involved in 364 (94.8\%) patients.

Majority of patients $(54.9 \%)$ at the time of diagnosis presented with T3 tumours. Macroscopically, the tumour size at presentation ranged from 1 to $18 \mathrm{~cm}$ in diameter with a median size of $6 \mathrm{~cm}$. Two hundred and two $(52.6 \%)$ patients had tumours above $6 \mathrm{~cm}$ in diameter. Lymph node metastasis at the time of diagnosis was reported in $272(70.8 \%)$ patients and most of them $(45.3 \%)$ had N3 pathologic nodal status. Distant metastasis at presentation was recorded in $82(21.4 \%)$ patients and occurred mostly in the lungs, liver, bone and brain in 46 (56.1\%), $23(28.0 \%), 20(24.4 \%)$ and $8(9.8 \%)$ patients, respectively. Most of patients presented with stage III disease $(63.0 \%)$ followed by stage IV (21.4\%). Invasive ductal carcinoma was the 
most frequent histopathological type accounting for $91.7 \%$ of cases. Most patients $(63.8 \%)$ had poorly differentiated tumour (G3 grade) (Table 2). The presence of necrosis within the tumour was reported in $218(56.8 \%)$ patients.

Table 2: Clinicopathological pattern of breast cancer patients at BMC

\begin{tabular}{|c|c|c|c|}
\hline Variable & Response & No. patients & $\%$ \\
\hline \multirow{4}{*}{$\begin{array}{l}\text { Duration of symptoms (in } \\
\text { months) }\end{array}$} & $1-6$ & 52 & 13.5 \\
\hline & $6-12$ & 209 & 54.4 \\
\hline & $12-24$ & 78 & 20.3 \\
\hline & $>24$ & 45 & 11.7 \\
\hline \multirow[t]{3}{*}{ Laterality } & Right & 198 & 51.6 \\
\hline & Left & 180 & 46.9 \\
\hline & Bilateral & 6 & 1.5 \\
\hline \multirow[t]{6}{*}{ Anatomical quadrant } & Upper outer quadrant & 364 & 94.8 \\
\hline & Upper inner quadrant & 7 & 1.8 \\
\hline & Lower outer quadrant & 7 & 1.8 \\
\hline & Lower inner quadrant & 3 & 0.8 \\
\hline & Nipple & 2 & 0.5 \\
\hline & $>$ one quadrant & 134 & 34.9 \\
\hline \multirow[t]{3}{*}{ Metastasis } & Lymph node metastasis & 272 & 70.8 \\
\hline & Distant metastasis & 82 & 21.4 \\
\hline & Not reported & 45 & 11.7 \\
\hline \multirow[t]{5}{*}{ Primary tumour status } & T0 (No tumour detected) & 1 & 0.3 \\
\hline & T1(Tumour size $<2 \mathrm{~cm}$ ) & 36 & 9.4 \\
\hline & T2(Tumour size 2-5 cm) & 52 & 13.5 \\
\hline & $\mathrm{T} 3$ (Tumour size $>5 \mathrm{~cm}$ & 211 & 54.9 \\
\hline & $\begin{array}{l}\text { T4 (Tumour of any size with skin, chest } \\
\text { wall involvement) }\end{array}$ & 84 & 21.9 \\
\hline \multirow[t]{4}{*}{ Pathologic nodal status } & N0(no nodal involvement) & 94 & 24.5 \\
\hline & N1(ipsilateral, axillary nodes, mobile & 104 & 27.1 \\
\hline & N2 (ipsilateral axillary nodes, fixed) & 174 & 45.3 \\
\hline & N3(ipsilateral internal mammary nodes) & 12 & 3.1 \\
\hline \multirow[t]{5}{*}{ Clinical stage } & Stage 0 & 2 & 0.5 \\
\hline & Stage I & 18 & 4.7 \\
\hline & Stage II & 42 & 10.9 \\
\hline & Stage III & 242 & 63.0 \\
\hline & Stage IV & 82 & 21.4 \\
\hline \multirow[t]{5}{*}{ Histopathological type } & Invasive ductal carcinoma & 352 & 91.7 \\
\hline & Invasive lobular carcinoma & 13 & 3.4 \\
\hline & Mucinous carcinoma & 12 & 3.1 \\
\hline & Ductal carcinoma in situ & 2 & 0.5 \\
\hline & Other histological type & 5 & 1.3 \\
\hline \multirow[t]{3}{*}{ Histological grade } & G1(well differentiated) & 8 & 2.1 \\
\hline & G2 (moderate differentiated) & 131 & 34.1 \\
\hline & G3 (poorly differentiated) & 245 & 63.8 \\
\hline
\end{tabular}


Patients with tumour size greater than $6 \mathrm{~cm}$ (median tumour size) had significantly high rate of lymph node metastasis $(P=0.001)$ and presence of necrosis within the tumour $(P=0.012)$ compared to patients with tumour size less than $6 \mathrm{~cm}$ in diameter. Patients younger than 45 years (which is the median age) had significantly high rate of lymph node metastasis compared to the patients above the median age $(P=0.011)$.

\section{Treatment modalities}

Surgery, mainly mastectomy, was the main modality of treatment in these patients. A total of $382(99.5 \%)$ patients had mastectomy \pm axillary lymph node dissection. Two patients had an inoperable tumour because it was hard and fixed to the chest wall. None of the patients had breast conservation surgery (Table 3).

Table 3: The types of surgical procedures performed among patients with breast cancer at BMC (N= 382)

\begin{tabular}{lll}
\hline Types of surgical procedures & $\begin{array}{l}\text { Number of } \\
\text { patients }\end{array}$ & Percentage \\
\hline Modified radical mastectomy \pm Axillary lymph node dissection & 56 & 14.7 \\
Simple mastectomy \pm Axillary lymph node dissection & 242 & 63.4 \\
Toilet mastectomy \pm Axillary lymph node dissection & 84 & 22.0 \\
\hline
\end{tabular}

The use of chemotherapy was documented in $172(44.8 \%)$ patients. Of these, $154(89.5 \%)$ were used as adjuvant therapy and in the remaining $18(10.5 \%)$ patients chemotherapy was used as neo-adjuvant therapy in some patients with grade IIIb to down stage the tumour. The drug combinations were CMF-cyclophosphamide, methotrexate, 5-fluorouracil and CAFcyclophosphamide, adriamycin and 5-fluorouracil. These drugs were given parenterally and repeated at 3-4 weekly intervals for 6 courses. Only 53 (30.8\%) patients completed the courses. Hormonal therapy using Tamoxifen was prescribed to all patients. Adjuvant radiotherapy required in all patients was reported in only $45(11.7 \%)$ patients. No patient had immunotherapy in this study.

\section{Follow up of patients}

Follow-up visits were scheduled every 3 months for 2 years and every six months thereafter for a period of five years or death. Follow-up of patients ranged from 3 to 62 months with a median of 12 months. At the end of five year follow up period, only 124 (32.3\%) patients were available for follow up and the remaining $260(65.0 \%)$ patients were either lost to follow up or died. Of those who were available for follow up, 92 (74.2\%) patients were tumour free at one year. At the end of five years of follow up, only 84 patients were still alive giving an overall five-year survival rate of $21.8 \%$. The 5-year survival rate for patients with early disease was higher than for those with advanced disease $(P=0.000)$. According to multivariate logistic regression analysis; age of patient at diagnosis $(P=0.002)$, stage of disease $(P=0.001)$, extent of lymph node involvement $(P=0.012)$ and histological grade $(P=0.004)$ were found to be independent predictors of overall survival. Local recurrence of cancer was reported in $22(17.7 \%)$ patients and this was significantly related to the stage of disease $(P=0.003)$ and non-adherent to adjuvant therapy $(P=0.021)$. 


\section{Discussion}

Breast cancer management has been a major challenge to surgeons in developing countries, principally due to the lack of advanced technology and late presentation of patients to the hospital (Kene et al., 2010). In this study, the median age of patients was 45 years which is in agreement with other studies in developing countries (Kene et al., 2010; Rambau et al., 2011), but lower than the median age reported in western countries where the median age at diagnosis is in the sixth decade of life (Ikpatt et al., 2002; Fregene \& Newman, 2005; Awadelkarim et al., 2008). In African women, the diagnosis of breast cancer is often made between 35 and 45 years of age. This is approximately 10-15 years earlier than peak incidence for western countries (Vorobiof et al., 2001; Adesunkanmi et al., 2006; Rambau et al., 2011). The reasons for the early age at onset of breast cancer among black women are poorly understood but could probably be connected to the aggressive nature of the disease (Chlebowski et al., 2005; Kene et al., 2010).While numerous theories have been proposed to explain this age difference, including age at menarche, time of first delivery, parity, socio-demographic factors, body mass index, and underlying genetic difference, none are completely satisfactory and more research is needed in this area (Vorobiof et al., 2001; Omar et al., 2003; Adesunkanmi et al., 2006). It has been reported that the occurrence of breast cancer at young age is associated with a worst prognosis and thus prognosis improves with age, with the best prognosis in patients over 75 years (Zavagno et al, 2000; Rambau et al., 2011). In our study, patients aged above 60 years accounted for only $4.2 \%$ of cases.

In the present study, more than half of the patients were multiparous which is contrary to the belief that multiparity is associated with decreased risk of developing breast cancer. These would have been the reverse since the higher the number of full term pregnancies, the greater the protection from breast cancer and that there is a reduction in risk of breast cancer by $7 \%$ for each birth after the first, in the absence of breast feeding. Also women who breast feed reduce their risk compared to those who do not (Adebamowo \& Ajayi, 2000). We could not establish the reason for this observation.

The proportion of patients this study that was premenopausal was $63.8 \%$, while $36.2 \%$ were postmenopausal. This is in contrast with observations in the developed countries where premenopausal patients accounted for less than one-third of the patients (Adelusola et al., 1996). Adebamowo et al. (1999) reported a much higher incidence of $80 \%$ of premenopausal women in Nigeria breast cancer patients. The reason for high incidence of premenopausal women in the present study may be attributed to the fact that the majority of patients in our study were young in their reproductive age group.

This study showed that most of the patients presented late with advanced disease commonly with clinical Stage III and IV. The findings are similar to several other centres in developing countries (Amir et al., 1996; Adebamowo \& Ajayi, 2000; Montazeri et al, 2003; Calleb, 2006; Kene et al., 2010). Similar findings have also been reported by Rambau et al. (2011) at the same centre. These findings are at variant with what is reported in developed countries where most of patients presents early with early stage of the disease (Parkin et al., 2005). Late presentation and advanced disease at the time of diagnosis in most developing countries may be explained by delay in seeking medical services, poor breast cancer awareness, poor referral 
system and medical service, lack of screening programmes and natural aggressive biological behaviour of tumour (Amir et al., 1996; Parkin et al., 2005; Rambau et al., 2011). The authors could not establish the reasons for the late presentation in the present study. This calls for further study to explain this observation. In order to reduce the late presentation of breast cancer the importance of early diagnosis should be emphasized through adequate information dissemination. There is therefore need for the establishment of standard breast cancer screening programme including installation of mammographic machine in many hospitals.

In this study, the right breast was mostly affected with the upper outer quadrant mostly involved either singly or in combination with other parts of the breast. This is in contrast to previous studies conducted in other centres where the left breast was reported to be the most commonly affected (Odigie et al., 2003; Adesunkanmi et al., 2006; Kene et al., 2010). The upper outer quadrant is also reported to be commonly involved in a West Africa study (Odigie et al., 2003). This may probably be due to the association of the upper outer quadrant with the axillary tail which is a channel for drainage of lymph to the axillary lymph nodes and may carry micrometastasis more than the other quadrants of the breast. The reason for the right breast predilection is not well understood.

Invasive ductal carcinoma was the commonest histological type of breast cancer in our study and most of patients had poorly differentiated tumour. This is consistent with reports from many centres (Odigie et al, 2003; Ekanem \& Aligbe, 2006). This tumour commonly metastasizes to the axillary lymph nodes and the prognosis is poor than that for the other types (Harns et al., 1992). As reported by Rambau et al. (2011), the tumour size in this study was significantly associated with younger age (below 45 years) at presentation, high rate of lymph node metastasis, high histological grade tumours and presence of necrosis within the tumour. The association between tumour size and younger age at presentation could explain the reasons for aggressive disease at younger age.

In the present study, more than two thirds of patients had lymph node metastasis at the time of diagnosis and about one-fifth of cases had distant metastasis to the lungs, liver, bone and brain, the rate which is higher than that reported in other studies (Amir et al., 1996; Calleb, 2006; Kene et al., 2010; Rambau et al, 2011). High lymph node and distant metastasis in our study is due to the fact that most patients in the present study present late when the disease is already in advanced stages. In developing countries, most patients are already in advanced stages of disease at the time of diagnosis of breast cancer, which has been proven both in the present study and in literature (Calleb, 2006; Kene et al., 2010; Rambau et al., 2011). The high rate of lymph node metastasis seen in our patients could also be accounted for by the large tumour size at the time of diagnosis.

The treatment of breast cancer requires multidisciplinary approach (Guadagnoli et al., 1998; Cardoso et al., 2003). Treatment modalities of breast cancer include surgery, chemotherapy, radiotherapy, hormonal and immunotherapy. Surgery is considered the primary treatment for breast cancer, with many early stage patients being cured with surgery alone. The goals of breast cancer surgery include the complete resection of the primary tumour, with negative margins to reduce the risk of local recurrences, and pathologic staging of the tumour and axillary lymph nodes to provide necessary prognostic information. In keeping with other studies (Vorobiof et al., 2001; Omar et al., 2003; Adesunkanmi et al., 2006; Calleb, 2006), surgery 
was the most common modality of breast cancer treatment in this study. Modified radical, simple and toilet mastectomy with or without axillary clearance were offered to those whose lesions were still operable. None of our patients had breast conservation surgery. This is a reflection of the advanced nature of breast cancer in our patients. Few that presented with early stages were not considered for conservation surgery largely because of non-availability of radiotherapy facilities at BMC. These patients were treated with modified radical mastectomy. Simple and toilet mastectomy with or without axillary clearance were offered to patients with late stages (stage III and IV). Breast conservation surgery is currently the most popular treatment for breast cancer, representing $75-80 \%$ of all operations (Veronesi et al., 2005). Unfortunately, advanced stages at presentation and poor infrastructure for treatment of breast cancer have made this mode of surgical treatment less popular in many developing countries including Tanzania. Breast reconstruction after mastectomy for breast cancer has become a standard procedure. None of the patients was offered plastic surgery for breast reconstruction following mastectomy due to late presentation and the necessity for prolonged follow-up before the reconstructive procedure.

Combination chemotherapy given as either neo-adjuvant or adjuvant therapy is an important component of breast cancer treatment and it is widely used in many centres across the world (Ibrahimu et al., 2011). In the present study, only $44.8 \%$ of patients received chemotherapy and $69.2 \%$ among those offered chemotherapy defaulted and did not complete the course. This observation is in keeping with other African studies (Kene et al., 2011; Ibrahimu et al., 2011). Non adherence to chemotherapy is a major challenge in breast cancer treatment especially in resource poor settings. Reasons for non-adherence in most developing countries include financial difficulty, relatively feeling well after commencement of chemotherapy, resorting to alternative treatment and drug side effects (Lawal \& Adesunkanmi, 2008; Ibrahimu et al., 2011). We could not establish the reasons for non-adherence to chemotherapy in our study due to the retrospective nature of the study.

Tamoxifen is the only hormonal therapy currently approved for adjuvant therapy in patients with breast cancer (Sweetland, 2004). Tamoxifen alone reduces risk of recurrence and improves overall survival in all age groups in oestrogen positive cancers in both premenopausal and post menopausal women (Calleb, 2006; Gakwaya et al., 2008). Facilities for oestrogen receptor assay at BMC are not available. All our patients are given tamoxifen, once a histological diagnosis is established irrespective of menstrual status.

Adjuvant radiotherapy is an integral part of the management of breast cancer, particularly in patients with large tumours and many positive nodes (Overgaard, 2001). In this study, only $11.7 \%$ of patients requiring adjuvant radiotherapy had access to this modality of treatment. Adjuvant radiotherapy is required to reduce the risk of local recurrence following surgery (Gakwaya et al., 2008). Failure to access to this modality of treatment in our patients can be explained by the fact that radiotherapy is not available at BMC and therefore patients requiring this form of treatment had to travel long distances to receive radiotherapy elsewhere. Because of lack of funds at the time of referral for radiotherapy in the majority of patients, only less than $20 \%$ of patients were able to travel and received this form of treatment.

Locoregional recurrence of breast cancer occurs in 5\% to $40 \%$ of patients whose primary disease was initially managed by mastectomy, and up to one third of these patients 
will have concomitant distant organ metastases (Chagpar et al., 2003; Buchanan et al., 2006). In this study, local recurrence of cancer was reported in $17.7 \%$ of cases, a figure which is higher than that reported in other studies (Schmoor et al., 2000; Buchanan et al., 2006). The reasons for high rate of local recurrence in the present study may be attributed to advanced stage of cancer at the time of diagnosis and non-adherence to adjuvant treatment.

The overall survival rate of $21.8 \%$ is significantly low compared to the survival rate of breast cancer patients managed in developed countries which is $95 \%$ and above (Smigal et al, 2006).This poor outcome is similar to that report by Anyanwu (2000) in Nigeria. This perhaps could be due to late presentation because of ignorance and lack of screening services. In our patients, the factors that significantly affect prognosis were age of the patient, stage of disease, axillary lymph node status and histological tumour grade. Tumour stage and axillary nodal status have consistently been shown to be the most important independent predictors of overall survival (Park et al, 2011).

The potential limitation of this study is the fact that information about some patients was incomplete in view of the retrospective nature of the study. This might have introduced some bias in our findings. In addition, follow-up was poor and irregular and so it was difficult to know the exact time of recurrence or death. Large number of loss to follow up was also a potential limitation of this study as this may have underestimated the local recurrence and overall survival rates. However, despite these limitations, findings from this study provide local data that can be utilized to improve the care of breast cancer in our local setting.

In conclusion, our study indicate that breast cancer patients in this region present late with advanced disease and high rate of lymph node metastasis and show a trend towards relative young age at diagnosis. Poor breast cancer awareness, poor referral system, lack of screening programs and natural aggressive biological behaviour of tumour may contribute to advanced disease at the time of diagnosis. There is need to improve public enlightenment of breast cancer and set up screening centres to encourage early presentations.

\section{Acknowledgements}

We are very grateful to all the Senior House Officer in the Department of Surgery for their support in data collection. We also appreciate the contribution of all the staff members of the medical records department, histopathology laboratory, theatre and surgical wards for the support they provided during data collection.

\section{References}

Adebamowo, C.A. \& Adekunle, O.O. (1999) Case control study of the epidemiological risk factors of breast cancer in Nigeria. British Journal of Surgery 86, 665-668.

Adebamowo, C.A. \& Ajayi, O.O. (2000) Breast cancer in Nigeria. West African Journal of Medicine 19, 179-191.

Adelusola, K., Fadiran, O.A., Adesunkanmi, A.R.K. \& Odesanmi, W.O. (1996) Breast cancer in Nigerian women. Niger Medical Practice 31, 17-29. 
Adesunkanmi, A.R., Lawal, O.O., Adelusola, K.A. \& Durosimi, M.A. (2006) The severity, outcome and challenges of breast cancer in Nigeria. Breast 15, 399-409.

Amir, H., Kwesigabo, G., Aziz, M.R. \& Kitinya, J.N. (1996) Breast cancer and conservative surgery in sub Saharan Africa. East African Medical Journal 73, 81-82.

Anyanwu, S.N. (2000) Survival following treatment of primary breast cancer in eastern Nigeria. East African Medical Journal 77, 539-543.

Awadelkarim, K.D., Arrizi, C. \& Elamin, E.O.M. (2008) Pathological, clinical and prognostic characteristics of breast cancer in Central Sudan versus Northern Italy: implications for breast cancer in Africa. Histopathology 52, 445-456.

Buchanan, C.L., Dorn, P.L., Fey, J., Giron, G., Naik, A., Mendez, J. \& Murphy, C. (2006) Locoregional recurrence after mastectomy: incidence and outcomes. Journal of American College of Surgeons 203, 469-474.

Cardoso, F. \& Piccart, M.J. (2003) The best use of chemotherapy in the adjuvant setting. Breast $12,522-528$.

Chagpar, A., Meric-Bernstam, F. \& Hunt, K.K. (2003) Chest wall recurrence after mastectomy does not always portend a dismal outcome. Annals of Surgical Oncology 10, 628-634.

Chlebowski, R.T., Chen, Z., Anderson, G.L., Rohan, T., Aragaki, A. \& Lane, D. (2005) Ethnicity and breast cancer: factors influencing differences in incidence and outcome. Journal of National Cancer Institute 97, 439-448.

Elston, C.W. \& Ellis, O. (1991) Pathological prognostic factors in breast cancer. The value of histological grade in breast cancer: Experience from a large study with long-term follow-up. Histopathology 19, 403-410.

Elston, C.W. (2005) Classification and grading of invasive breast carcinoma. Verh Dtsch Ges Pathologie 89, 35-44

Ekanem, V.J. \& Aligbe, J.U. (2006) Histopathological types of breast cancer in Nigerian women: a 12-year review (1993-2004). African Journal of Reproductive Health 10, 71-75

Elumelu, T.N., Adenipekun, A.A., Abdus-salam, A.A. \& Bojude, A.D. (2011) Pattern of breast cancer metastasis at the Radiotherapy Clinic, Ibadan - A ten year review. Journal of American Science 7, 906-912.

Fregene, A. \& Newman, LA. (2005) Breast cancer in sub-Saharan Africa: how does it relate to a breast cancer in African-American women? Cancer 103, 1540-1550.

Guadagnoli, E., Shapiro, C.L., Weeks, J.C., Gurwitz, J.H., Borbas, C., Soumerai, S.B. (1998) The quality of care for treatment of early stage breast carcinoma: is it consistent with national guidelines? Cancer 83, 302-309.

Harns, J.R., Lippman, M.E., Veronesi, U. \& Millett, W. (1992) Breast cancer. New England Journal of Medicine 327, 473.

Henson, D.E., Ries, L., Freedman, L.S. \& Carriaga, M. (1991) Relationship among outcome, stage of disease, and histologic Grade for 22,616 Cases of Breast Cancer. The Basis for a Prognostic Index. Cancer 68, 2142-2149.

Hisham, A.N. \& Yip, C.H. (2004) Overview of breast cancer in Malaysian women: a problem with late diagnosis. Asian Journal of Surgery 27, 130-133. 
Ibrahim, N.A., Popoola, A.O., Oludara, M.A., Omodele, F.O. \& Fadeyibi, I.O. (2011) Breast cancer among urban Nigerian women: Appraising presentation and the quality of care. Macedonian Journal of Medical Sciences 4, 388-392.

Ikpatt, O.F., Kuopoo, I., Ndoma-Egbe, R. \& Collen, Y. (2002) Breast cancer in Nigeria and Finland: epidemiological, clinical and histological comparison. Anticancer Research 22, 3005-3012.

Kene, T.S., Odigie, V.I., Yusufu, L.M.D., Shehu, S.M. \& Kase, J.T. (2010) Pattern of presentation and survival of breast cancer in a Teaching Hospital in North Western Nigeria. Oman Medical Journal 25, 104-107.

Lawal, A.O. \& Adesunkanmi, A.R. (2008) Paradox of wellness and non-adherence among Nigerian women on breast cancer chemotherapy. Journal of Cancer Research and Therapeutics 4, 107-110.

Montazeri, A., Ebrahimi, M., Mehrdad, N., Ansari, M., Sajadian, A. (2003) Delayed presentation in breast cancer: A study in Iranian women. BMC Womens Health. 3, 4

Ngoma, T.A. \& Mtango, D. (2002) The management of cancer patients at the Ocean Road Cancer Institute: Fifteen years experience. Tanzania Health Research Bulletin 4, 11-18.

Ngoma, T.A. \& Ngoma, M. (2004) Breast cancer and self-examination knowledge among Tanzanian women: implications for breast cancer health education. Tanzania Health Research Bulletin 6, 64-68.

Odigie, V.I., Yusufu, L.M., Rafindadi, A. \& da Rocha-Afodu, J.T. (2003) Breast cancer in Zaria. Nigerian Journal of Surgery 9, 46-50.

Ohanaka, C.E. \& Ofoegbu, R.O. (2002) The pattern of surgical cancers in Nigeria: the Benin experience. Tropical Doctor 32, 38-39.

Okobia, M.N. \& Osime, U. (2001) Clinicopathological study of carcinoma of the breast in Benin City. Africa Journal of Reproductive Health 5, 56-62.

Okobia, M.N., Bunker, C.H., Okonofua, F.E. \& Osimi, U. (2006) Knowledge, attitude and practice of Nigerian women towards breast cancer: A cross-sectional study. World Journal of Surgical Oncology 4, 1-9.

Omar, S., Khaled, H., Gaafar, R., Zekry, AR., Eissa, S. \& el-Khatib, O. (2003) Breast cancer in Egypt: a review of disease presentation and detection strategies. Eastern Mediterranean Health Journal 9, 448-463.

Overgaard, M. (2001) Radiotherapy as a part of a multidisciplinary treatment strategy in early breast cancer. European Journal of Cancer 37, S33-S43.

Park, Y., Kitahara, T., Takagi, R. \& Kato, R. (2011) Current status of therapy for breast cancer worldwide and in Japan. World Journal of Clinical Oncology 10,125-134.

Parkin, D.M. (2003) Cancer in Africa: Epidemiology and Prevention. IARC Publications No. 153.

Parkin, DM., Bray, F., Ferlay, J. \& Pisani, P. (2005) Global cancer statistics. Cancer Journal for Clinicians 55, 74-108.

Rambau, P.F., Chalya, P.L., Manyama, M.M. \& Kahima, J.K. (2011) Pathological features of Breast Cancer seen in Northwestern Tanzania: a nine years retrospective study. BMC Research Notes 4, 214.

Sant, M., Allemani, C., Berrino, F., Coleman, MP., Aareleid, T., Chaplain, G. \& Gafa, L (2004). Breast carcinoma survival in Europe and the United. States. Cancer 100, 715-722. 
Schmoor, C., Sauerbrei, W., Bastert, G. \& Schumacher, M. (2000) Role of isolated locoregional recurrence of breast cancer: results of four prospective studies. Journal of Clinical Oncology 18, 1696.

Smigal, C., Jemal, A., Ward, E., Cokkinides, V., Smith, R., Howe, H.L. \& Thun, M. (2006) Trends in breast cancer by race and ethnicity. Cancer Journal for Clinicians 56, 168-183.

Sweetland, H.M. (2004) Adjuvant therapy for breast cancer. Surgery International 66, 161-164.

Veronesi, U., Boyle, P., Goldhirsch, A, Orecchia, R. \& Viale, G. (2005) Breast cancer. Lancet 365, 1727-1741.

Vorobiof, D.A., Sitas, F. \& Vorobiof, G. (2001) Breast cancer incidence in South Africa. Journal of Clinical Oncology 15, 125S-7S.

Zavagno, G., Meggiolaro, F., Pluchinotta, A., Bozza, F. \& Favretti, F. (2000) Influence of age and menopausal status on pathologic and biologic features of breast cancer. Breast 9, 320328.

Zhou, J. \& Zhong, Y. (2004) Breast cancer immunotherapy. Cellular \& Molecular Immunology 1, 247-255. 